\title{
Physical Ingredients Controlling Stability and Structural Selection of Empty Viral Capsids
}

\author{
María Aznar and David Reguera*
}

Statistical and Interdisciplinary Physics Section, Departament de Física de la Matèria

Condensada, Universitat de Barcelona, Martí i Franquès 1, 08028-Barcelona, Spain

E-mail: dreguera@ub.edu 


\section{SUPPLEMENTARY MATERIAL}

\section{Calculation of the Expected Value of $\nu$ for Different Struc-}

\section{tures}

The theoretical values of the preferred angle that are expected to be optimal for each $\mathrm{T}$ number structure are calculated in this supplementary section. These values, denoted as $\nu_{\text {opt }}$, are calculated as the angle between nearest-neighbour capsomers obtained from the coordinates of the icosahedral spherical code listed in Ref. ${ }^{1}$ corresponding to the solution of the maximum volume problem. For a spherical capsid of radius $R$ centered at the origin of coordinates, the optimal angle is the angle formed between the vector $\vec{\Omega}_{1}=\frac{1}{R}\left(x_{1}, y_{1}, z_{1}\right)$ defining the orientation of a capsomer and the vector $\mathbf{r}_{12}=\left(x_{2}-x_{1}, y_{2}-y_{1}, z_{2}-z_{1}\right)$ connecting two neighbour capsomers, denoted by the subscripts 1 and 2. As shown in Fig. S1, by simple geometry:

$$
R \sin (\nu-\pi / 2)=\frac{1}{2} r_{i j}
$$

where $r_{12}=\left|\mathbf{r}_{1 \mathbf{2}}\right|=\sqrt{\left(x_{2}-x_{1}\right)^{2}+\left(y_{2}-y_{1}\right)^{2}+\left(z_{2}-z_{1}\right)^{2}}$ and 1 , and 2 refer to two nearestneighbours capsomers. The coordinates in Ref. ${ }^{1}$ are given for a unit sphere of radius $R=1$. Accordingly:

$$
\nu_{\text {opt }}=\frac{\pi}{2}+\arcsin \left(r_{12} / 2\right)
$$

The value of $\nu_{\text {opt }}$ can be calculated using two adjacent hexamers or a pentamer and a nearest-neighbour hexamer. Table S1 shows the values of $\nu_{\text {opt }}$ calculated from eq. S2 for the smallest T-number structures and the snub cube, in both cases. 


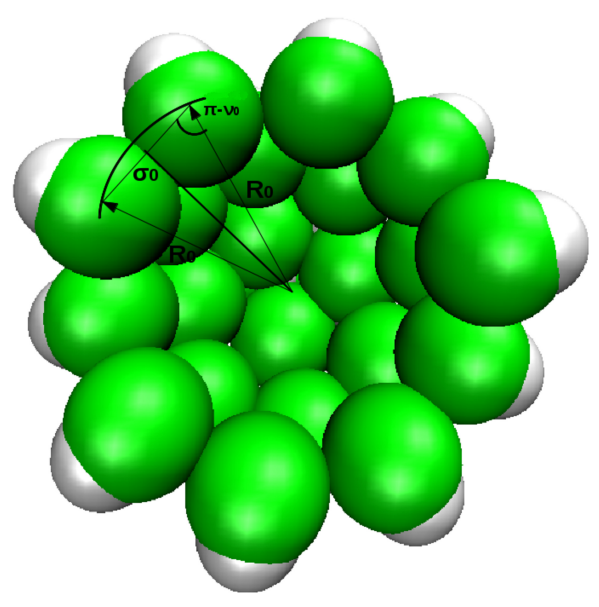

Figure S1: Transverse section of $\mathrm{T}=3$ shell of radius $R_{0}$ and preferred angle $\nu_{0}$.

Table S1: Optimal values of the preferred angle $\nu_{o p t}$ predicted for the lowest T-number structures and the snub cube, calculated between two hexamers, $\nu_{o p t}^{h h}$, (or 2 pentamers in the $\mathbf{T}=1$ case) and between a hexamer and a pentamer, $\nu_{o p t}^{h p}$.

\begin{tabular}{ccc}
\hline Structure & $\nu_{o p t}^{h h}$ & $\nu_{o p t}^{h p}$ \\
\hline $\mathrm{T}=1$ & 2.124 & \\
Snub cube & 1.954 & 1.912 \\
$\mathrm{~T}=3$ & 1.936 & 1.897 \\
$\mathrm{~T}=4$ & 1.885 & 1.847 \\
$\mathrm{~T}=7$ & 1.797 & 1.780 \\
\hline
\end{tabular}

\section{Local and Global Bending Stiffness}

The effective macroscopic bending stiffness $\kappa$ can be calculated in terms of the local parameters of the interaction potential by comparing the global bending energy in the continuum limit with that obtained from our model. For simplicity, this comparison will be performed for the bending energy of a cylinder in our coarse-grained model.

Let us assume a hexagonally-ordered network of coarse-grained capsomers on the surface of a cylinder of length $L$ and radius $R$, see Fig. S2 (a). According to this figure, the positions 


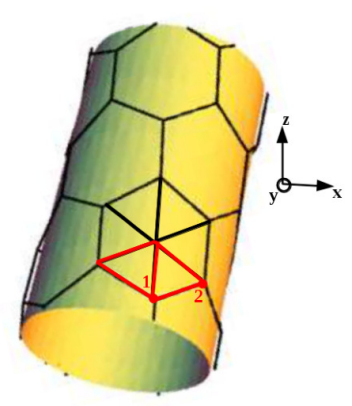

(a)

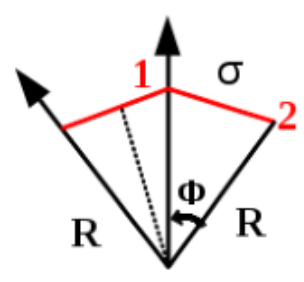

(b)

Figure S2: (a) Hexagonal lattice of capsomers placed over a cylinder of radius $R$ and length $L$ and (b) cross-section of the positions of capsomers 1 and 2 over the cylinder.

of capsomer 1 and 2 are given by:

$$
\begin{aligned}
& x_{1}=0 ; y_{1}=R ; z_{1}=0 \\
& x_{2}=R \sin (\phi) ; y_{2}=R \cos (\phi) ; z_{2}=\frac{\sigma}{2}
\end{aligned}
$$

where the origin of $z$ has been taken at the location of capsomer 1 . The distance between these two capsomers is $r_{12}^{2}=R^{2} \sin ^{2}(\phi)+R^{2}(\cos (\phi)-1)^{2}+\frac{\sigma^{2}}{4}$, that at equilibrium will coincide with the equilibrium distance $\sigma$, thus $r_{12}^{2}=\sigma^{2}$. By regrouping this equation we obtain $2 R^{2}(1-\cos (\phi))=\frac{3}{4} \sigma^{2}$, that using the approximation for small angles $\cos (\phi) \approx 1-\frac{\phi^{2}}{2}$, finally yields

$$
\phi=\frac{\sqrt{3}}{2} \frac{\sigma}{R}
$$

According to Eq. 7the bending energy between capsomer 1 and 2 around the equilibrium distance and orientation is given by

$$
E_{b}^{12}=2 \frac{\epsilon_{0}}{2 \alpha^{2}}\left(\theta_{12}-\frac{\pi}{2}\right)^{2}
$$


where $\cos \theta_{i j}=\hat{r}_{i j} \cdot \vec{\Omega}_{i}$. Using the positions of capsomers 1 and 2 from eq. S3, one obtains

$$
\begin{aligned}
\cos \theta_{12}= & \frac{1}{\sigma}\left(R \sin (\phi), R(\cos (\phi)-1), \frac{\sigma}{2}\right) \cdot(0,1,0)= \\
& =\frac{R}{\sigma}(\cos (\phi)-1) \approx \frac{-R \phi^{2}}{2 \sigma}
\end{aligned}
$$

For small angles $\arccos (x) \approx \frac{\pi}{2}-x$ and the angle between capsomer 1 and 2 then becomes

$$
\theta_{12}=\frac{\pi}{2}+\frac{R \phi^{2}}{2 \sigma}
$$

Using eq. $\mathrm{S} 6$ in eq. S5 the total contribution between 1 and 2 is

$$
E_{b}=\frac{9}{64} \frac{\epsilon_{0}}{\alpha^{2}} \frac{\sigma^{2}}{R^{2}}
$$

Each capsomer interacts with 4 neighbours. Thus, the bending energy per capsomer becomes $E_{b}^{c}=\frac{4}{2} E_{b}$, where we have divided by 2 to avoid double counting. Now if we multiply $E_{b}^{c}$ by the total number of capsomers in the cylinder, given by $N_{c}=\frac{2 \pi R L}{\frac{\sqrt{3}}{2} \sigma^{2}}$, (where $A_{H}=\frac{\sqrt{3}}{2} \sigma^{2}$ is the effective area of a capsomer) the total bending energy is:

$$
E_{b}^{t o t}=\frac{4}{2} N_{c} E_{b}=\frac{3 \sqrt{3}}{8} \pi \frac{\epsilon_{0}}{\alpha^{2}} \frac{L}{R}
$$

Comparing eq. S9 with the standard expression of the total bending energy of a cylinder ${ }^{2}$ $E^{c y l}=\pi \kappa \frac{L}{R}$, one finally gets the effective bending stiffness

$$
\kappa=\frac{3 \sqrt{3}}{8} \frac{\epsilon_{0}}{\alpha^{2}} .
$$




\section{References}

(1) Hardin, R.; Sloane, N.; Smith, W. Tables of Spherical Codes with Icosahedral Symmetry, published electronically at http://neilsloane.com/icosahedral.codes/.

(2) Lidmar, J.; Mirny, L.; Nelson, D. Virus Shapes and Buckling Transitions in Spherical Shells. Phys. Rev. E 2003, 68, 051910. 